\title{
Nanotechnology Impact Towards Innovative Biomedical Engineering
}

\author{
Makoto Kikuchi
}

National Defense Medical College Research Institute / Department of Medical Engineering, National Defense Medical College, JAPAN

\begin{abstract}
:
Nanotechnology is emerging as a new field enabling the creation and application of materials, devices, and systems at atomic and molecular levels and the exploitation of novel properties that emerge at the nanometer scale. Many areas of biomedical engineering are expected to benefit from nanotechnology including sensors for use in the laboratory, the clinic, and within the human body, new formulations and routes for drug delivery, and biocompatible, high-performance materials for use in implants. Examples of potential uses of nanotechnology in biomedical engineering include the early detection and treatment of disease. Recently, enormous researchers with this notice have been launched to encourage the applications for research, in the general areas of nanoscience and nanotechnology as related to

1) The development of new research tools for elucidating biological principles essential for the design and implementation of nanostructured materials for use in biomedical engineering, and

2) The transfer of nanotechnology advances in other fields of science and engineering to develop new ways to help prevent, detect, diagnose, and treat disease and disorders.
\end{abstract}

Nanostructures offer a new paradigm for materials manufacture by submicron-scale assembly (ideally, utilizing self-organization and self-assembly) to create entities from the "bottom up" rather than the "top down" ultraminiaturization method of chiseling smaller structures from larger ones. Each significant advance in understanding the physical/chemical bio properties and fabrication principles, as well as in development of predictive methods to control them, is likely to lead to major advances in our ability to design, fabricate and assemble the nanostructures and nonodevice into a working system being applicable to healthcare technology. 